\title{
HOMOLOGICAL DIMENSION AND CARDINALITY
}

\author{
BY \\ B. L. OSOFSKY
}

\begin{abstract}
Let $\{e(i) \mid i \in \mathscr{I}\}$ be an infinite set of commuting idempotents in a ring $R$ with 1 such that

$$
\prod_{\alpha=0}^{n} e\left(i_{\alpha}\right) \prod_{\beta=n+1}^{m}\left(1-e\left(i_{\beta}\right)\right) \neq 0
$$

for $\left\{i_{\alpha} \mid 0 \leqq \alpha \leqq n\right\} \cap\left\{i_{\beta} \mid n+1 \leqq \beta \leqq m\right\}=\varnothing$. Let $I$ be the right ideal generated by these idempotents. We show that the projective dimension of $I$ is $n<\infty$ if and only if the cardinality of $I=\boldsymbol{\aleph}_{n}$. As a consequence, a countable direct product of fields has global dimension $k+1$ if and only if $2 \boldsymbol{x}_{0}=\boldsymbol{\aleph}_{k}$. The same is true for a full linear ring on a countable dimensional vector space over a field of cardinality at most $2 N_{0}$. On the other hand, if $2 \aleph_{0}>\boldsymbol{\aleph}_{\omega}$, then any right and left self-injective ring which is not semiperfect, any ring containing an infinite direct product of subrings, any ring containing the endomorphism ring of a countable direct sum of modules, and many quotient rings of such rings must all have infinite global dimension.
\end{abstract}

This paper continues the investigation of the relationship between homological dimension and cardinality questions started in [4] and [5], combining the techniques of $\S 7$ of [5] with a modification of a projective resolution in Pierce [7]. Employing a result of Hausdorff and Tarski, we show that the $\aleph$ corresponding to $2 \aleph_{0}$ plays an important role in the global dimension of rings where one can find analogues of characteristic functions of subsets of a set of orthogonal idempotents.

1. Homological dimension of an ideal generated by commuting idempotents. We first calculate the homological dimension of a right ideal of a ring $R$ (with 1) generated by a "nice" set of idempotents, and then show that several types of rings possess such idempötents.

A family $\mathfrak{A}=\{e(i) \mid i \in \mathscr{I}\}$ of idempotents of $R$ is called nice if

(i) $e(i) e(j)=e(j) e(i) \forall i, j \in \mathscr{I}$.

(ii) $\prod_{\alpha=1}^{n} e\left(i_{\alpha}\right) \prod_{\beta=n+1}^{m}\left(1-e\left(i_{\beta}\right)\right) \neq 0$ if

$$
\left\{i_{\alpha} \mid 1 \leqq \alpha \leqq n\right\} \cap\left\{i_{\beta} \mid n+1 \leqq \beta \leqq m\right\}=\varnothing .
$$

For any nice family of idempotents $\mathfrak{A}$, define $I_{\mathfrak{A}}=\sum_{e \in \mathfrak{A}} e R$. Assume $\mathfrak{A}$ is indexed by a linearly ordered set $\mathscr{I}$. Let

$$
P_{n}(\mathfrak{U})=\underset{i_{0}<i_{1}<\cdots<i_{n}}{\bigoplus}\left\langle i_{0}, \ldots, i_{n}\right\rangle R \subseteq R^{\mathscr{\sigma}^{n}}
$$

Received by the editors December 1, 1969.

AMS Subject Classifications. Primary 1690; Secondary 0430, 1640.

Key Words and Phrases. Homological dimension, global dimension of rings, cardinal number corresponding to $2 \aleph$, direct products of rings, endomorphism rings.

Copyright (C) 1970, American Mathematical Society 
where $\left\langle i_{0}, \ldots, i_{n}\right\rangle$ represents that function in $R^{\mathscr{F}^{n}}$ which takes the value 0 everywhere except at $\left(i_{0}, \ldots, i_{n}\right) \in \mathscr{I}^{n}$ where it takes the value $\prod_{\alpha=0}^{n} e\left(i_{\alpha}\right)$. We observe that

and for any $i \in \mathscr{I}$,

$$
\left\langle i_{0}, \ldots, i_{n}\right\rangle \prod_{\alpha=0}^{n} e\left(i_{\alpha}\right)=\left\langle i_{0}, \ldots, i_{n}\right\rangle
$$

(*) $\quad P_{n}(\mathfrak{X})=\left[\underset{i_{0}<\cdots<i_{n}}{\bigoplus}\left\langle i_{0}, \ldots, i_{n}\right\rangle e(i) R\right] \oplus\left[\underset{i_{0}<\cdots<i_{n}}{\bigoplus}\left\langle i_{0}, \ldots, i_{n}\right\rangle(1-e(i)) R\right]$.

We call the first summand $e(i) P_{n}(\mathfrak{A})$ and the second $(1-e(i)) P_{n}(\mathfrak{A})$. We rewrite the boundary operator of [7] to avoid using characteristic 2 ; namely, define

$$
\begin{array}{rlrl}
d_{0}: P_{0}(\mathfrak{U}) & \rightarrow I_{\mathfrak{U}}, & d_{0}\left\langle i_{0}\right\rangle & =e\left(i_{0}\right), \\
d_{n}: P_{n}(\mathfrak{U}) \rightarrow P_{n-1}(\mathfrak{U}), & d_{n}\left\langle i_{0}, \ldots, i_{n}\right\rangle & =\sum_{\alpha=0}^{n}(-1)^{\alpha}\left\langle i_{0}, \ldots, \hat{l}_{\alpha}, \ldots, i_{n}\right\rangle\left(e\left(i_{\alpha}\right)\right)
\end{array}
$$

where $\hat{\imath}_{\alpha}$ means delete $i_{\alpha}$.

Proposition 1.

$$
\mathscr{P}(\mathfrak{U}): \cdots \stackrel{d_{n+1}}{\longrightarrow} P_{n}(\mathfrak{U}) \stackrel{d_{n}}{\longrightarrow} \cdots \stackrel{d_{1}}{\longrightarrow} P_{0}(\mathfrak{U}) \stackrel{d_{0}}{\longrightarrow} I_{\mathfrak{A}} \longrightarrow 0
$$

is a projective resolution of $I_{\mathfrak{U}}$.

Proof. $P_{n}(\mathfrak{A})$ is projective since it is isomorphic to a direct sum of projective right ideals.

That $\mathscr{P}(\mathfrak{A})$ is a complex is a standard computation. Every term in

$$
d_{n-1} d_{n}\left\langle i_{0}, \ldots, i_{n}\right\rangle
$$

appears twice with opposite signs.

$$
d_{0} d_{1}\left\langle i_{0}, i_{1}\right\rangle=d_{0}\left(\left\langle i_{1}\right\rangle e\left(i_{0}\right) e\left(i_{1}\right)-\left\langle i_{0}\right\rangle e\left(i_{0}\right) e\left(i_{1}\right)\right)=e\left(i_{0}\right) e\left(i_{1}\right)-e\left(i_{0}\right) e\left(i_{1}\right)=0 .
$$

Let $d_{n} p=0, p=\sum_{\alpha=1}^{m}\left\langle i_{0, \alpha}, \ldots, i_{n, \alpha}\right\rangle r_{\alpha}$. Let $i$ be the largest $i_{n, \alpha}$ such that $\left\langle i_{0, \alpha}, \ldots, i_{n, \alpha}\right\rangle r_{\alpha} \neq 0$, and let $e(i) p$ and $(1-e(i)) p$ be the projections of $p$ on the appropriate summands of $(*)$. Since

$$
d_{n}\left(e(i) P_{n}(\mathfrak{U})\right) \subseteq e(i) P_{n-1}(\mathfrak{U}) \quad \text { and } \quad d_{n}\left((1-e(i)) P_{n}(\mathfrak{U})\right) \subseteq(1-e(i)) P_{n-1}(\mathfrak{U}),
$$

$d_{n} e(i) p=d_{n}(1-e(i)) p=0$. A straightforward calculation shows that

$$
d_{n+1}\left(\sum_{i_{n, \alpha} \neq i}\left\langle i_{0, \alpha}, \ldots, i_{n, \alpha}, i\right\rangle e(i) r_{\alpha}\right)-(-1)^{n+1} e(i) p=q \in \bigoplus\left\langle i_{0}, \ldots, i_{n-1}, i\right\rangle R .
$$

Since $d_{n} q=0$, looking at terms of $d_{n} q$ not involving $i$ shows that $q$ must $=0$. We observe that $(1-e(i)) p$ has fewer than $m$ nonzero terms since $i$ is actually equal to some $i_{n, \alpha}$ in a nonzero term of $p$, and then use induction on $m$ to get

$$
(1-e(i)) p \in d_{n+1} P_{n+1}(\mathfrak{U}) .
$$

Hence $\mathscr{P}(\mathfrak{A})$ is exact. 
Let hd $\left(I_{\mathfrak{A}}\right)$ denote the homological dimension of $I_{\mathfrak{A}}$, that is, the smallest $k$ such that $d_{k} P_{k}(\mathfrak{Q})$ is projective, or $\infty$ if no such $k$ exists.

Proposition 2. Let card $(\mathscr{I})=\boldsymbol{\aleph}_{\Omega}$, hd $\left(I_{\mathfrak{U}}\right) \leqq k<\infty$. Then if $k<\Omega$, there exists a set $\mathscr{J} \subseteq \mathscr{I}$ such that card $(\mathscr{J})=\aleph_{k}$ and $d_{k} P_{k}(\{e(i) \mid i \in \mathscr{J}\})$ is a direct summand of $d_{k} P_{k}(\mathfrak{U})$.

Proof. This is identical with the proof of Proposition 5.2(c) of [5].

Proposition 3. Let $\mathscr{I}$ be an ordinal such that for some $n \in \omega$, no ordinal of cardinality $<\aleph_{n}$ is cofinal in $\mathscr{I}$. Then $\mathrm{hd}\left(I_{\mathfrak{A}}\right) \geqq n$.

Proof. If $n=0$, then there is nothing to prove, so we may assume $n \geqq 1$. Assume hd $\left(I_{\mathfrak{Q}}\right)=k<n$. We will use induction on $k$.

If $k=0, I_{\mathfrak{A}}$ is projective. By Proposition 2 (which uses the snaking argument of Kaplansky [3]), there exists a countable set $\mathscr{J} \subseteq \mathscr{I}$ such that

$$
I_{\mathfrak{A}}=\sum_{j \in \mathscr{J}} e(j) R \oplus I^{\prime} .
$$

Let $i^{\prime} \in \mathscr{I}-\mathscr{J}$. Then $e\left(i^{\prime}\right)=a+b, a \in \sum_{j \in \mathscr{I}} e(j) R, b \in I^{\prime}$. Let $a=\sum_{\alpha=1}^{m} e\left(j_{\alpha}\right) r_{\alpha}$. Since $\mathfrak{U}$ is a nice set of idempotents, for any $j^{\prime} \in \mathscr{J}-\left\{j_{\alpha} \mid 1 \leqq \alpha \leqq m\right\}$,

$$
\bar{a}=e\left(i^{\prime}\right) e\left(j^{\prime}\right) \prod_{\alpha=1}^{m}\left(1-e\left(j_{\alpha}\right)\right) \neq 0 .
$$

But

$$
\begin{aligned}
(a+b) \bar{a} & =e\left(i^{\prime}\right) \bar{a}=\bar{a}=\bar{a} e\left(i^{\prime}\right)=\bar{a}(a+b) \\
& =\bar{a} b \in e\left(j^{\prime}\right) R \cap e\left(i^{\prime}\right) R \subseteq a R .
\end{aligned}
$$

Hence $b \bar{a}=0$. But $\bar{a}^{2}=\bar{a}=(\bar{a} b)(\bar{a} b)=0$, a contradiction.

Now assume $k \geqq 1$ and $k<n$. By Proposition 2, there exists $\mathscr{J} \subseteq \mathscr{I}$ with card $(\mathscr{J})$ $=\aleph_{n-1}$ and $d_{k} P_{k}(\{e(i) \mid i \in \mathscr{J}\})$ is a direct summand of $d_{k} P_{k}(\mathfrak{U})$. Since $k<n$, by hypothesis $\tau=\sup (\mathscr{J})+1<\mathscr{I}$. Now

$$
\begin{aligned}
& P_{k-1}(\mathfrak{U})=\bigoplus_{\left\{J_{\alpha}\right\} \subseteq \mathscr{J}}\left\langle j_{0}, \ldots, j_{k-1}\right\rangle e(\tau) R \\
& \oplus \underset{\left\{j_{\alpha}\right\} \subseteq \mathscr{J}}{\oplus}\left\langle j_{0}, \ldots, j_{k-1}\right\rangle(1-e(\tau)) R \\
& \oplus \underset{\left\{i_{\alpha}\right\} \mp \mathscr{J}}{\oplus}\left\langle i_{0}, \ldots, i_{k-1}\right\rangle R \\
& =\bigoplus_{\left\{j_{\alpha}\right\} \subseteq \mathscr{J}} d_{k}\left\langle j_{0}, \ldots, j_{k-1}, \tau\right\rangle R \\
& \oplus \underset{\left\{j_{\alpha}\right\} \subseteq \mathcal{J}}{\oplus}\left\langle j_{0}, \ldots, j_{k-1}\right\rangle(1-e(\tau)) R \\
& \oplus \underset{\left\{i_{\alpha}\right\} \nsubseteq \mathscr{J}}{\oplus}\left\langle i_{0}, \ldots, i_{k-1}\right\rangle R
\end{aligned}
$$

and

$$
d_{k} P_{k}(\mathfrak{U})=d_{k} P_{k}(\{e(i) \mid i \in \mathscr{J}\}) \oplus K .
$$


Then

$$
e(\tau) d_{k} P_{k}(\mathfrak{U})=e(\tau) d_{k} P_{k}(\{e(i) \mid i \in \mathscr{J}\}) \oplus e(\tau) K \cap K
$$

where premultiplication by $e(\tau)$ indicates as before the appropriate projection in (*). Now $e(\tau) d_{k} P_{k}(\{e(i) \mid i \in \mathscr{J}\})$ is a direct summand of $e(\tau) d_{k} P_{k}(\mathfrak{U})$ and $M=$

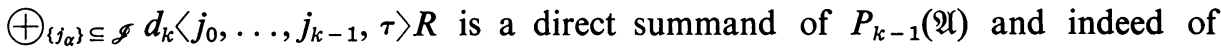
$e(\tau) P_{k-1}(\mathfrak{A})$. Moreover, $M \supseteq e(\tau) d_{k} P_{k}(\{e(i) \mid i \in \mathscr{J}\})$ by the proof used for exactness of $\mathscr{P}(\mathfrak{U})$. Hence $e(\tau) d_{k} P_{k}(\{e(i) \mid i \in \mathscr{J}\})$ is actually a direct summand of $e(\tau) P_{k-1}(\mathfrak{U})$ since a direct summand of a direct summand of a module is a direct summand of the entire module.

Define $\mathfrak{B}=\{e(\tau) e(i) \mid i \in \mathscr{J}\}$. Then $\mathfrak{B}$ is a nice set of idempotents of $R$ since $\mathfrak{A}$ is and since $1-e(\tau) e(i)=1-e(\tau)+e(\tau)(1-e(i))$. Moreover, the complex $\mathscr{P}(\mathfrak{B})$ is naturally isomorphic to $e(\tau) P(\{e(i) \mid i \in \mathscr{J}\})$ in an obvious manner. In particular, kernel $_{\mathscr{P}(\mathfrak{B})} d_{k-1}$ is a direct summand of $P_{k-1}(\mathfrak{B})$, so hd $\left(I_{\mathfrak{B}}\right) \leqq k-1$. By the induction hypothesis, $n-1 \leqq$ hd $\left(I_{\mathfrak{B}}\right) \leqq k-1$, contradicting $k<n$.

Proposition 4. If $\mathscr{I}$ is a set such that card $(\mathscr{I})=\aleph_{n}$, then hd $\left(I_{\mathfrak{A}}\right) \leqq n$.

Proof. If $I$ is countable, order it by $\omega$. Then

$$
\begin{gathered}
I_{\mathfrak{A}}=e(0) R \oplus e(1)(1-e(0)) R \oplus e(2)(1-e(0))(1-e(1)) R \\
\oplus \cdots \oplus e(n) \prod_{\alpha=1}^{n-1}(1-e(\alpha)) R \oplus \cdots
\end{gathered}
$$

is projective.

Assume the proposition for all nice sets of idempotents of cardinality less than $\aleph_{n}, n \geqq 1$. Index $\mathfrak{A}$ by the first ordinal of cardinality $\boldsymbol{\aleph}_{n}$. Then $I_{\mathfrak{A}}$ is a well-ordered ascending union of subideals of homological dimension $\leqq n-1$, so hd $\left(I_{\mathfrak{A}}\right) \leqq n$ as in [1].

Propositions 3 and 4 combined with some obvious set theoretic computations such as those in [4] show

THEOREM A. If $\mathfrak{A}$ is a nice set of idempotents, then hd $\left(I_{\mathfrak{A}}\right)=n$ if and only if $\operatorname{card}(\mathfrak{U})=\boldsymbol{\aleph}_{n}$.

2. Rings possessing nice sets of idempotents. Theorem $A$ is of interest mainly because several "natural" rings possess rather "large" nice sets of idempotents. Of course, Pierce's free Boolean rings, or indeed the analogous free algebra generated by commuting idempotents over any ring will have nice sets of idempotents. In this section we use a result in [8] to get a method of constructing nice sets of idempotents in other kinds of rings.

Proposition 5. Let $\{\varepsilon(k) \mid k \in \mathscr{K}\}$ be an infinite set of orthogonal idempotents of $R$ with card $(K)=\aleph$. Assume for each $\mathscr{L} \subseteq \mathscr{K}$ there exists an idempotent $e(\mathscr{L}) \in R$ such that

$$
\varepsilon(k) e(\mathscr{L})=e(\mathscr{L}) \varepsilon(k)=\varepsilon(k) \chi_{L}(k)
$$


where $\chi_{L}$ denotes the characteristic function of $L$.

$$
e(\mathscr{L}) e(\mathscr{M})=e(\mathscr{M}) e(\mathscr{L}) \text { for all } \mathscr{L}, \mathscr{M} \subseteq \mathscr{K} \text {. }
$$

Then there exists a nice set of idempotents $\mathfrak{A} \subseteq R$ such that card $(\mathfrak{U})=2^{\aleph}$.

Proof. Hausdorff and Tarski have shown that there exists a family $\mathfrak{F}$ of subsets of $\mathscr{K}$ such that card $(\mathfrak{F})=2^{N}$ and for any disjoint finite subsets $U$ and $V$ of $\mathfrak{F}$, $\bigcap_{\mathscr{L} \in U} \mathscr{L} \cap \bigcap_{\mathscr{M} \in V}(\mathscr{K}-\mathscr{M}) \neq \varnothing$. (For a proof see Sikorski [8, p. 45].) Then $\{e(\mathscr{L}) \mid \mathscr{L} \in \mathfrak{F}\}$ is a nice set of idempotents of $R$, for if $U$ and $V$ are disjoint finite subsets of $\mathfrak{F}$ and $k \in \bigcap_{\mathscr{L}_{\in U}} \mathscr{L} \cap \bigcap_{\mathscr{M} \in V}(\mathscr{K}-\mathscr{M})$, then

$$
\varepsilon(k) \prod_{\mathscr{L} \in U} e(\mathscr{L}) \prod_{\mathscr{M} \in V}(1-e(\mathscr{M}))=\varepsilon(k) \neq 0 .
$$

What kinds of rings satisfy the hypotheses of Proposition 5 ? Clearly $\prod_{k \in \mathscr{K}} R_{k}$ or $\mathrm{Hom}_{S}\left(\oplus_{k \in \mathscr{K}} M_{k}, \bigoplus_{k \in \mathscr{K}} M_{k}\right)$ do, where the $R_{k}$ are rings and the $M_{k}$ are modules over a ring $S$. In these cases the $\varepsilon(k)$ and $e(\mathscr{L})$ are appropriate projections. Also, if $S$ satisfies the hypotheses of Proposition 5, so does any over-ring of $S$.

Proposition 6. Let $R$ be a ring such that for some set $U \subseteq\left\{e R \mid e^{2}=e \in R\right\}$, the elements of $U$ form a complete, complemented lattice under join $=+$ and meet $=\cap$. Let $\left\{\varepsilon^{\prime}(k) \mid k \in \mathscr{K}\right\}$ be an infinite set of orthogonal idempotents of $R$ such that $\varepsilon^{\prime}(k) R \in U$ for all $k \in \mathscr{K}$. Then there exists $\{e(\mathscr{L}) \mid \mathscr{L} \subseteq \mathscr{K}\}$ satisfying the hypotheses of Proposition 5, where $\varepsilon(k)=e(\{k\})$.

Proof. By hypothesis, $\sup \left\{\varepsilon^{\prime}(k) R \mid k \in \mathscr{K}\right\}=e R$ for some $e=e^{2} \in R$. Let $\mathscr{L} \subseteq \mathscr{K}$. Then

$$
e R=\sup \left\{\varepsilon^{\prime}(k) R \mid k \in \mathscr{L}\right\} \oplus \sup \left\{\varepsilon^{\prime}(k) R \mid k \notin \mathscr{L}\right\}
$$

since $e R$ contains each supremum on the right, their sum contains $\left\{\varepsilon^{\prime}(k) R \mid k \in \mathscr{K}\right\}$, and their intersection is an idempotent generated right ideal in $U$ containing no $\varepsilon^{\prime}(k)$ for $k \in \mathscr{K}$. Let $e(\mathscr{L})$ be the projection of $e$ on $\sup \left\{\varepsilon^{\prime}(k) R \mid k \in \mathscr{L}\right\}$ with respect to this decomposition. One readily verifies that $e(\mathscr{L}) e(\mathscr{M})=e(\mathscr{M}) e(\mathscr{L})=e(\mathscr{L} \cap \mathscr{M})$ by looking at the decomposition

$$
\begin{aligned}
e R= & \sup \left\{\varepsilon^{\prime}(k) R \mid k \in \mathscr{L} \cap \mathscr{M}\right\} \oplus \sup \left\{\varepsilon^{\prime}(k) R \mid k \in \mathscr{L}-\mathscr{M}\right\} \\
& \oplus \sup \left\{\varepsilon^{\prime}(k) R \mid k \in \mathscr{M}-\mathscr{L}\right\} \oplus \sup \left\{\varepsilon^{\prime}(k) R \mid k \in \mathscr{K}-\mathscr{L} \cup \mathscr{M}\right\}
\end{aligned}
$$

and the result follows.

Proposition 7. Let $R$ be a right self-injective ring such that either $R$ is regular in the sense of von Neumann or $R$ is left self-injective or no $x \in R$ annihilates $\bigoplus_{k \in \mathscr{X}} \varepsilon(k) R$ on the left, where $\{\varepsilon(k) \mid k \in \mathscr{K}\}$ is some set of orthogonal idempotents. Then $R$ satisfies the hypotheses of Proposition 5 for any right ideal generated by orthogonal idempotents in the first two cases, and for the right ideal generated by the given set in the third case. 
Proof. We will reduce the first two cases to the third. Let $\left\{\varepsilon^{\prime}(k) \mid k \in \mathscr{K}\right\}$ be a set of orthogonal idempotents of $R, e R=$ an injective hull of $\oplus \varepsilon^{\prime}(k) R$. Let $\varepsilon(k)$ $=\varepsilon^{\prime}(k) e$. Then $\varepsilon(k) R=\varepsilon^{\prime}(k) R$ and $\{\varepsilon(k) \mid k \in \mathscr{K}\} \cup\{1-e\}$ is a set of orthogonal idempotents. Since $I=\oplus \varepsilon(k) R \oplus(1-e) R$ is essential in $R_{R}$, if $R$ is regular it is well known that the left annihilator of $I$ is zero. If $R$ is left self-injective, let $R f$ be an injective hull of $\oplus R \varepsilon(k) \oplus R(1-e), f=f^{2}$. Then the map

$$
\Pi_{r}: R \rightarrow \prod_{k \in \mathscr{K}} \varepsilon(k) R \times(1-e) R
$$

given by $\Pi_{r}(x)=(\langle\varepsilon(k) x\rangle,(1-e) x)$ must be monic since its kernel has zero intersection with an essential submodule of $R$. Since $\Pi(1-f)=0, f=1$. Then the left analogue of $\Pi_{r}$ has kernel zero, which is precisely the third situation.

Now let $\{\varepsilon(k) \mid k \in \mathscr{K}\}$ be a set of orthogonal idempotents in $R$ such that the left annihilator of $\oplus \varepsilon(k) R=0$. Let $E_{1}$ and $E_{2}$ be two injective hulls of $\bigoplus_{k \in \mathscr{L}} \varepsilon(k) R$ for $\mathscr{L} \subseteq \mathscr{K}$. Then $R=E_{1} \oplus F_{1}=E_{2} \oplus F_{2}$ where each $F_{i}$ is an injective hull of

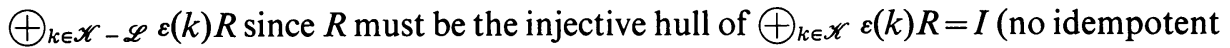
$1-f$ annihilates $I$ ). Let $1=e_{i}+f_{i}, i=1,2$, be the corresponding representations of 1 . Then $\left(e_{1}-e_{2}\right) \varepsilon(k)=0$ for all $k \in \mathscr{K}$, so $e_{1}=e_{2}$ and $E_{1}=E_{2}$. Hence the set of (idempotent generated) injective hulls of ideals $\bigoplus_{k \in \mathscr{L}} \varepsilon(k) R, \mathscr{L} \subseteq \mathscr{K}$, forms a complete complemented lattice and we may apply Proposition 5.

3. Conclusions. In this section we list some corollaries to the results in $\S \S 1$ and 2 , and insert a few remarks on the results.

3.1. Let $R$ be a ring possessing a nice set of idempotents of cardinality $\geqq \boldsymbol{\aleph}_{\omega}$. Then $R$ has infinite global dimension. Examples of such rings are endomorphism rings of direct sums of at least $\boldsymbol{\aleph}_{\omega}$ modules, direct products of at least $\boldsymbol{\aleph}_{\omega}$ rings, any nonzero quotient of a full linear ring on an $\boldsymbol{\aleph} \geqq \boldsymbol{\aleph}_{\omega}$ dimensional vector space, and two-sided self-injective rings containing sets of orthogonal idempotents of cardinality $\boldsymbol{\aleph}_{k}$ for each $k \in \omega$.

3.2. If $2 \aleph_{0}>\boldsymbol{N}_{\omega}$, then any infinite direct product of rings, endomorphism ring of an infinite direct sum of modules, or nonsemiperfect two-sided injective ring must have infinite global dimension. Moreover, if $I$ is an ideal of $R=$ one of the above types of rings, and if there exists an infinite set of orthogonal idempotents of $R$ none of which is in $I$, then $R / I$ has infinite global dimension. We note that if $R$ is regular or if $R$ is self-injective, then if $R / I$ is not semiperfect, it will have a countable set of orthogonal idempotents generating a right ideal $J^{\prime}$, and the preimage $J$ of $J^{\prime}$ in $R$ will contain a countable set of orthogonal idempotents $\left\{e_{i} \mid i \in \omega\right\}$ such that $J=\sum_{i=0}^{\infty} e_{i} R+I$. Thus $R / I$ will have global dimension $=\infty$.

3.3. If the $\boldsymbol{\aleph}_{k}$ corresponding to $2^{\aleph_{0}}$ is less than $\boldsymbol{\aleph}_{\omega}$, the global dimension of a countable direct product of fields is equal to $k+1$. We thus have a homological dimension statement equivalent to the continuum hypothesis similar to that found in [5].

3.4. By looking at quotients of injective modules in [6], we showed that the kinds of rings mentioned in the above remarks could not be hereditary. The main 
theorem of that note had hypotheses requiring that for a given set of orthogonal idempotents $\left\{e_{i} \mid i \in \mathscr{I}\right\}$ and $\mathscr{A} \subseteq \mathscr{I}$, there exist $m_{\mathscr{A}} \in R$ such that $e_{i} m_{\mathscr{A}}=0$ for all $i \notin \mathscr{A}$, and $m_{\mathscr{A}} e_{i}=e_{i}$ for all $i \in \mathscr{A}$. If, in addition, we require that $\left\{m_{\mathscr{A}}\right\}$ be commuting idempotents, these hypotheses will give rise to nice sets of orthogonal idempotents as in $\$ 2$ and thus show that such rings cannot be hereditary by looking at projective resolutions.

3.5. It is an open question whether the hypotheses of Proposition 6 can be weakened to $R$ is one-sided self-injective. Commutativity of nice sets of idempotents was used strongly in getting the projective resolution of $\S 1$, and just selecting any injective hull in a ring will not guarantee commutativity without some kind of uniqueness property. Perhaps there is some way to select injective hulls corresponding to subsets of a set of orthogonal idempotents in such a way that commutativity is assured, but such a technique seems rather elusive at the moment.

3.6. If $2 \aleph_{0}=\aleph_{1}$, the results in $\S \S 1$ and 2 give a lower bound of 2 on the global dimension of a countable dimensional full linear ring. If the field is suitably small, this is the global dimension. However, if the field has cardinality $>\boldsymbol{N}_{1}$, an upper bound on the global dimension is not as easy to obtain. It appears to be an open question whether the global dimension of a full linear ring is indeed dependent on the cardinality of the field as well as the dimension of the vector space if one assumes the generalized continuum hypothesis. The problem is illustrated by the following:

Let $V$ be an $\boldsymbol{K}$-dimensional right vector space over a division ring $F$ of cardinality $b>2^{\aleph}$, and let $R=\operatorname{Hom}_{F}(V, V)$. Then $R$ has cardinality $b^{\aleph}=\aleph_{\alpha}$, and the global dimension of $R$ is at most $\alpha+1$. We will show that $R$ has a right ideal generated by $\aleph_{\alpha}$ but no fewer elements. All we can say about the homological dimension of this ideal is that it is $\leqq \alpha$. We also show that $R$ has a right ideal of homological dimension 1 generated by $b$ but no fewer elements, so ideals requiring many generators may still have low dimensions.

We use the following two known results (see [2, pp. 67-68]).

LEMMA 1 (ERDÖs-KAPLANSKY). There exists a set of sequences $\left\{\left\langle b_{j}^{(\gamma)}\right\rangle \mid j \in \omega, \gamma \in \Gamma\right\}$ such that the cardinality of $\Gamma$ is b and every $n \times n$ matrix $\left(b_{j}^{\left(\gamma_{i}\right)}\right)_{1 \leqq i, j \leqq n}$ is nonsingular.

LEMMA 2. $\operatorname{dim}_{F} F^{\aleph}=b^{\aleph}$.

Proposition. $R$ has a right ideal generated by $b^{*}$ but no fewer elements.

Proof. Since $V$ is a right vector space, right ideals of $R$ are completely determined by the lattice of ranges of elements in the ideal. Hence, we need only construct a set of $b^{*}$ subspaces of $V$ such that no one of them is contained in a finite sum of others in the set.

Let $\left\{b^{(\gamma)} \mid \gamma \in \Gamma\right\}$ be a basis for $F^{\aleph}$, where $b^{(\gamma)}=\left\langle b_{\alpha}^{(\gamma)}\right\rangle, \alpha \in \mathscr{A}, \mathscr{A}$ a set of cardinality §. By Lemma $2, \Gamma$ has cardinality $b^{\aleph}$. Also, let $\left\{e_{\alpha} \mid \alpha \in \mathscr{A}\right\}$ be a basis for $V$. 
Since $\boldsymbol{\aleph}^{n}=\boldsymbol{\aleph}$ for all $n \in \omega$ and $\boldsymbol{\aleph}_{0} \boldsymbol{N}=\boldsymbol{\aleph}$, we may express $\mathscr{A}$ as a disjoint union of subsets $\mathscr{A}_{\mathrm{f}}$ indexed by the set $\mathfrak{F}$ of all finite subsets of $\mathscr{A}$, where $\mathscr{A}_{f}$ has the same cardinality as $\mathfrak{f}$. We may also assume that each $\mathscr{A}_{\mathfrak{f}}$ is indexed by $\mathfrak{f}$, that is,

$$
\mathscr{A}_{\mathfrak{f}}=\{\alpha(\beta) \mid \beta \in \mathfrak{f}\} \text {. }
$$

Let $W_{\gamma}$ be the subspace of $V$ generated by

$$
\left\{\sum_{\beta \in \mathfrak{f}} e_{\alpha(\beta)} b_{\beta}^{(\gamma)} \mid \mathfrak{f} \in \mathfrak{F}\right\}
$$

Assume $W_{\delta} \subseteq W_{\gamma_{1}}+\cdots+W_{\gamma_{n}}$.

For each $\mathfrak{f} \in \mathfrak{F}$, every $W_{\gamma}$ contains precisely one vector in $\sum_{\alpha \in \mathscr{A}_{\mathfrak{f}}} e_{\alpha} F$. Hence $\operatorname{dim}\left(\sum_{i=1}^{n} W_{y_{\mathrm{f}}}\right) \cap \sum_{\alpha \in \mathscr{A}_{\mathrm{f}}} e_{\alpha} F \leqq n$. Let

$$
k=\operatorname{dim}\left(\sum_{i=1}^{n} W_{\gamma_{i}}\right) \cap \sum_{\alpha \in \mathscr{A}_{\mathfrak{g}}} e_{\alpha} F
$$

be the maximum dimension possible for $\mathfrak{g} \in \mathfrak{F}$, and assume

$$
\left\{\sum e_{\alpha(\beta)} b_{\beta}^{\left(y_{i}\right)} \mid \beta \in \mathfrak{g}, 1 \leqq i \leqq k\right\}
$$

are linearly independent. Let

$$
\sum_{\beta \in \mathfrak{g}} e_{\alpha(\beta)} b_{\beta}^{(\delta)}=\sum_{i=1}^{k}\left(\sum_{\beta \in \mathfrak{g}} e_{\alpha(\beta)} b_{\beta}^{\left(\gamma_{i}\right)}\right) x_{i}
$$

Then for all $\beta \in \mathfrak{g}, b_{\beta}^{(\delta)}=\sum_{i=1}^{k} b_{\beta}^{\left(\gamma_{i}\right)} x_{i}$.

Let $\beta^{\prime} \in \mathscr{A}-\mathfrak{g}, \mathfrak{h}=\mathfrak{g} \cup\left\{\beta^{\prime}\right\}$. Then

$$
\sum_{i=1}^{k}\left(\sum_{\beta \in \mathfrak{h}} e_{\alpha(\beta)} b_{\beta}^{\left(\gamma_{i}\right)}\right) x_{i}-\sum_{\beta \in \mathfrak{h}} e_{\alpha(\beta)} b_{\beta}^{(\delta)}=e_{\alpha\left(\beta^{\prime}\right)}\left(\sum_{i=1}^{k} b_{\beta^{\prime}}^{\left(y_{1}\right)} x_{i}-b_{\beta^{\prime}}^{(\delta)}\right),
$$

$\alpha\left(\beta^{\prime}\right) \in \mathscr{A}_{\mathfrak{h}}$.

By the maximality of $k$ and the independence of $\left\{\sum_{g} e_{\alpha(\beta)} b_{\beta}^{\left(y_{i}\right)} \mid 1 \leqq i \leqq k\right\}$,

$$
\sum_{i=1}^{k} b_{\beta^{\prime}}^{\left(\gamma_{i}\right)} x_{i}-b_{\beta^{\prime}}^{(\delta)}
$$

must be zero. But then $b^{(\delta)}=\sum_{i=1}^{k} b^{\left(y_{i}\right)} x_{i}$, a contradiction.

Proposition. $R$ has a right ideal of homological dimension 1 generated by $b$ but no fewer elements.

Proof. Let $\left\{\left\langle b_{j}^{(\gamma)}\right\rangle \mid j \in \omega, \gamma \in \Gamma\right\}$ be a set of sequences satisfying the properties asserted in Proposition 1. Let $\left\{e_{i} \mid i \in \omega\right\}$ be any linearly independent set of elements of $V$. Let $W_{\gamma}$ be the subspace spanned by

$$
\left\{\sum_{j=0}^{2^{n}-1} e_{2^{n}+j} b_{j}^{(\gamma)} \mid n \in \omega\right\}
$$


By the nonsingularity of $n \times n$ submatrices formed from $\left\{\left\langle b_{j}^{\gamma}\right\rangle\right\}, W_{\gamma} \cap \sum_{i=1}^{m} W_{\gamma_{i}}$ $\subseteq \sum_{j=1}^{2 l+1} e_{j} F$, where $2^{l}$ is the largest power of 2 which is less than or equal to $m$. In particular, a set of projections $E_{\gamma}$ onto $W_{\gamma}$ are independent modulo the socle $S$ of $R$. Then

$$
0 \rightarrow S \cap \sum E_{\gamma} R \rightarrow \sum E_{\gamma} R \rightarrow \bigoplus_{\gamma}\left(E_{\gamma} R+S / S\right) \rightarrow 0
$$

is exact, $S \cap \sum E_{\gamma} R$ is projective, $E_{\gamma} R+S / S \approx E_{\gamma} R /\left(S \cap E_{\gamma} R\right)$ has dimension 1, so $\sum E_{\gamma} R$ has dimension at most $1 . \sum E_{\gamma} R$ cannot be projective since it needs too many generators to be a direct sum of principal ideals (see [3]). Hence $\sum E_{\gamma} R$ has dimension precisely 1 .

3.7. Editorial. In this paper as well as in [5], statements on homolgical dimension were found to be equivalent to the continuum hypothesis. In these works, if $2 \aleph_{0} \neq \aleph_{1}$, then $\boldsymbol{\aleph}_{1}$ appears in the role of a stumbling block in getting from $\boldsymbol{\aleph}_{0}$ to $2 \aleph_{0}$. The "natural" structures all have cardinality $\aleph_{0}$ or $2 \aleph_{0}$ (or greater). There is no way in these papers to get one's hands on $\boldsymbol{\aleph}_{1}$. Such a situation is aesthetically (or intuitively if you prefer) repugnant to me. In addition, a finite full linear ring has global dimension $=0$. When one goes from finite to $\boldsymbol{\aleph}_{0}$, of course this changes, and in any case the global dimension goes up to at least 2 . However, a jump from zero to infinity is quite a jump and appears rather unintuitive. Moreover, infinite global dimension for nonsemiperfect two-sided injective rings is also very surprising. For those reasons, the hypothesis $2 \aleph_{0}=\boldsymbol{N}_{1}$ appears to me to be the natural one applying to the axiom system in which homological algebra is done, and $2 \boldsymbol{\aleph}_{0}>\boldsymbol{\aleph}_{\omega}$ has somewhat upsetting consequences.

\section{BIBLIOGRAPHY}

1. M. Auslander, On the dimension of modules and algebras. III: Global dimension, Nagoya Math. J. 9 (1955), 67-77. MR 17, 579.

2. N. Jacobson, Structure of rings, Amer. Math. Soc. Colloq. Publ., vol. 37, Amer. Math. Soc., Providence, R. I., 1964. MR 36 \#5158.

3. I. Kaplansky, Projective modules, Ann. of Math. (2) 68 (1958), 372-377. MR 20 \#6453.

4. B. Osofsky, Global dimension of valuation rings, Trans. Amer. Math. Soc. 127 (1967), 136-149. MR 34 \#5899.

5. - Homological dimension and the continuum hypothesis, Trans. Amer. Math. Soc. 132 (1968), 217-230. MR 37 \#205.

6. — Noninjective cyclic modules, Proc. Amer. Math. Soc. 19 (1968), 1383-1384. MR 38 \#185.

7. R. Pierce, The global dimension of Boolean rings, J. Algebra 7 (1967), 91-99. MR 37 \#5269.

8. R. Sikorski, Boolean algebras, 2nd ed., Ergebnisse der Mathematik und ihrer Grenzgebiete, Neue Folge, Band 25, Academic Press, New York and Springer-Verlag, Berlin and New York, 1964. MR 31 \#2178.

RUTGERS UNIVERSITY,

New Brunswick, New JeRSEY 08903 